\title{
ANP (Atrial Natriuretic Peptide) presence in the heart of a tunicate, Ciona intestinalis
}

\section{Luana Lipari'1, Aldo Gerbino', Diego Lipari², Elvira Vittoria Farina ${ }^{2}$}

Dipartimento di Biomedicina Sperimentale e Neuroscienze Cliniche, ${ }^{1}$ Sez. Istologia ed Embriologia, ${ }^{2}$ Sez. Anatomia Umana, Universitá di Palermo, Italy

\begin{abstract}
Atrial natriuretic peptide was found in the heart of vertebrates, we studied the ANP presence in the heart of Ciona intestinalis. This is animal is very important because of the its evolutionary position between invertebrates and vertebrates. ANP presence was only revealed in myoepithelial cells of the myocardium. Results suggest the hypothesis that ANP is present not only in the vertebrates but also in the invertebrates and in Ciona heart ANP might play a similar role like in the heart of vertebrates.
\end{abstract}

Key words: ANP, heart, tunicates, Ciona intestinalis

\section{Introduction}

Atrial natriuretic peptide (ANP) has been discovered in the specific granules in atrial myocardiocytes of the adult rats [5], successively ANP was found also in ventricles, conducting system and atrioventricular valves in the heart of many mammals (dog, rabbit, man) and non mammalian vertebrates such as fish [6] and amphibian [1].

The biochemical and physiologic studies demonstrated that ANP is involved to regulation of the bloodpressure by its natriuretic, vasodilatative and diuretic action, besides ANP presence was demonstrated also in other organs such as brain $[3,4]$, salivary glands [10], lung [9,10] kidney [8], of the numerous different animal species, therefore ANP is involved in homeostasis of the other body fluids.

In consideration of diffusion of the ANP, whose presence was demonstrated in different species of Vertebrates such as fishes, amphibians, in some reptiles, while ANP is not present in birds, we think that also ANP may be present in animal species less evolved such as Chordate; in particular we carry out a immunohistochemical study to verify if the ANP is present in the heart of tunicate, Ciona intestinalis. We choose this animal species because of the Tunicates, in

Correspondence: F.E. Vittoria, Dipartimento Medicina Sperimentale Sez Anatomia Umana, Via del Vespro 129,

90127 Palermo, Italy; e-mail: farina.elviravitt@tiscali.it the evolutionary progression between a relationship vertebrates and invertebrates, indeed the Tunicates present a dorsal cord nothocord only in larval life but it is absorbed in the adult.

\section{Material and methods}

Adult specimens of Ciona intestinalis collected from the Gulf of Palermo were used. Hearts were removed and immediately were fixed in Bouin's fluid, dehydrated and embedded in paraffin. Sections (7-10 $\mu \mathrm{m}$ thick) were prepared. Some sections were stained using $\mathrm{H}+\mathrm{E}$ or Azan's method, some sections were immunostained for ANP and detected with avidin biotin complex or streptoavidin system. Endogenous peroxidase was blocked by incubation with PBS containing $0.3 \%$ hydrogen peroxide for $5 \mathrm{~min}$ at room temperature. Following blockage of the specific binding by normal goat serum, the sections were treated with ANP polyclonal antibody raise in rabbit (Chemicon) at 1/600 and 1/800 dilutions in $0.05 \mathrm{M}$ Tris Buffer $\mathrm{pH} 7.2$ for 12 hours at $4^{\circ} \mathrm{C}$, subsequently the sections were washed in PBS (three times, 15 min each) The reaction was revealed by avidin-biotin or streptoavidin system (Ylem) binding was demonstrated by amino-ethyl-carbazole as substrate. The negative controls were those treated with non immunized antiserum or omission of the primary antiserum.

\section{Results}

The observations at light microscopy evidence that the heart of Ciona intestinalis is a tubular organ, it derives by invagination of the dorsal wall of the pericardial vesicle whose margins are fused to form a raphe, thus the pericardium and myocardium are concentric tubular vesicles $\mathrm{T}$ tube fold to U-like (Fig. 1). 

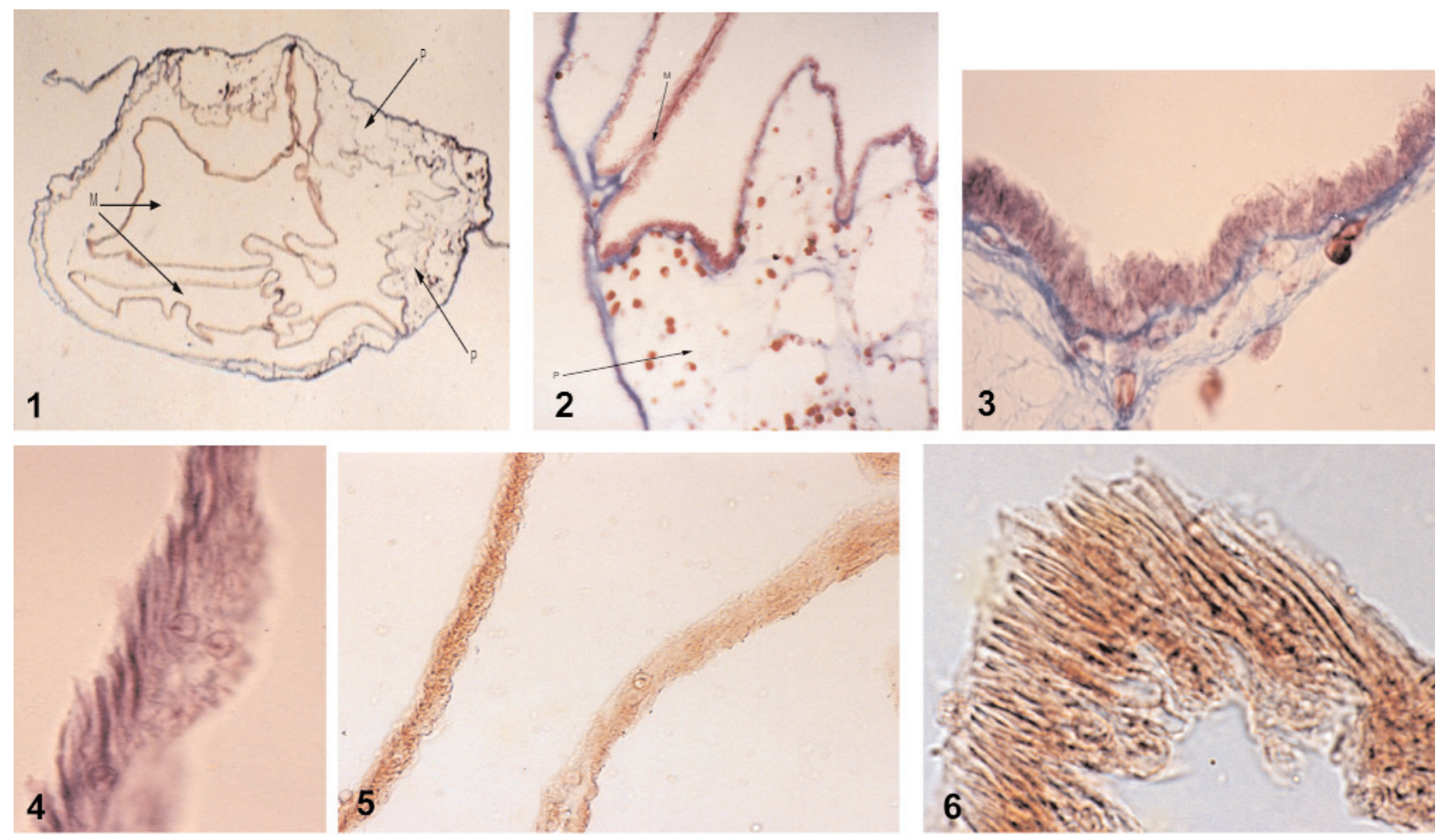

Fig. 1. Heart Ciona intestinalis. The heart presents the myocardium (M), are visible two inner tubules and the outer pericardium (P) (original magnification $\times 10)$. Fig. 2. Heart Ciona intestinalis. It is visible the invagination of the myocardium $(\mathrm{M})$. The pericardium $(\mathrm{P})$ with the two layer and a space between the layers (original magnification $\times 20$ ). Fig. 3. Heart Ciona intestinalis. Magnification of the pericardium (original magnification $\times 40$ ). Fig. 4. Heart Ciona intestinalis. In the myocardiocytes the nucleus is situated in the luminal area and the myofibrils are situated in the basal area of cell (original magnification $\times 63$ ). Fig. 5. Heart Ciona intestinalis. The ANPimmunopositive myocardium and ANP-immunonegative pericardium (original magnification $\times 20$ ). Fig. 6. Myocardium. The myocardiocytes show ANP- immunopositivity in the myofibrillar area of cells (original magnification $\times 40$ ).

The stained by Mallory-Azan's method sections show that the pericardium consists of two layers, whose the outer layer is connective tissue and the inner layer is an epithelium with flattened cells (Figs. 2,3). The myocardium is a tubular layer constituted of myocardiocytes. The myocardiocyte shows a apical area where the nucleus is located and a basal more intensely stained area where the myofibrils with a spiral arrangement are visible (Fig. 4).

The ANP-immunostained sections show that in the pericardium is immunonegative (Fig. 5) while in myocardium the myocardiocytes present a intense ANP-immunopositivity where is the myofibrillar component (Fig. 6).

\section{Discussion}

Ciona intestinalis is a member of the Tunicates a group of a sessile marine invertebrates. Despite appearances the Tunicates are member of own phylum, Cordata, their close evolutionary relationship to Vertebrates is most evident during developmental stages, the larvae present a dorsal notochord and a tail, upon the attachment to a substrate the tail and the cord regress. A recent morphological study of the heart of Ciona intestinalis was carried out by [2].

The results of our study show the ANP-immunostaining evidence that the pericardium is immunonegative indicating that in Ciona the pericardium is not involved in the ANP production, although in other species ANP was found in the pericardial fluid, but its cellular source was uncertain. In addition, a recent study in rat and human pericardial fluid demonstrated ANP-immunopositivity in mast-cells of the connective tissue [7]. The ANP immunopositivity in myocardiocytes demonstrated that also in Ciona ANP is present and that ANP might play the same role of the specific granules of different animal species.

Our results demonstrate that ANP is present also in the less evolved invertebrates and therefore ANP is a molecule that has undergone no or few changes during the evolutionary process.

ANP might play a similar function than it plays in other species and is involved, by still unknown mechanisms, in the regulation of the composition of fluids running in the heart and in the body lacunae, since in Ciona vessels do not present a continuous endothelium and an open type circulation occurs. 


\section{References}

[1] Caso VH, Paz D, Ruiz G. Differentiation of endocrine myocardiocytes in developing heart of the toad (Bufo arenarum Hensel). Int J Dev Biol. 1992;36:537-42.

[2] Davidson B. Ciona intestinalis as a model foer cardiac development. Semin Cell Dev Biol. 2007;18:16-26.

[3] Farina Lipari E, Lipari D, Dieli F, Valentino B. ANP secretion during development of the supraoptic nucleus. Eur J Histochem. 2005;49:379-384.

[4] Kawata M, Nakao K, Morii N, Kiso Y, Yamashita H, Imura H, Sano Y Atrial natriuretic polypeptide. Topographical distribution in the rat brain by radioimmunoassay and immunohistochemistry. Neuroscience. 1985a;16:521-46.

[ 5] Kish B. Electron microscopy of atrium of heart I. Guinea pig. Exp Med Surg. 1956;14:99-112.

[6] Johnson KR, Olson KR. Comparative physiology of the piscine natriuretic peptide system. Gen Comp Endocrinol. 2008;157:21-6.
[7] Martynova MG, Nakatseva EV, Emel'ianova MI, Moiseeva OM, Erokhina IL. Immunolocalization of ANP in mast cell of rat and human pericardium. Tsitologiia. 2008;50:237-42.

[8] Mc Kenzie JC, Scott J, Inagami T. Immunohistochemical localization of atrial natriuretic peptide in the developing and adult mammalian. Am J Anat. 1991;190:182-191.

[ 9] Perrault T and Gutkowska J. Role of atrial natriuretic factor in lung physiology and pathology. Am J Crit Care Med. 1995;1511:226-242.

[10] Valentino B, Farina Lipari E, Carini F, Valenza V. Immunohistochemical localization of atrial natriutretic factor (ANF) in the excretory system of rabbit parotid gland. Eur J Histochem. 1999; 43:241-245.

[11] Valentino B, Lipari D, Dieli F, Leone A, Farina Lipari E. Distribution of atrial natriuretic factor (ANF) in respiratory tract and lung showed by immunohistochemical and PCR Methods. Dent Med Probl. 2003;40:17-22.

Submitted: 1 July, 2009 Acceptted after reviews: 1 November, 2009 